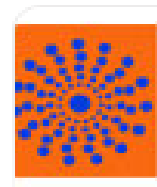

JOURNAL OF BEHA VIOR, HEALTH \& SOCIAL ISSUI

ints

Vázquez-Honorato, Luis Arturo; Salazar-Martínez, Bertha Lilia Arquitectura, vejez y calidad de vida. Satisfacción residencial y bienestar social Journal of Behavior, Health \& Social Issues, vol. 2, núm. 2, noviembre-abril, 2010, pp. 57-70 Asociación Mexicana de Comportamiento y Salud, A. C.

Distrito Federal, México 


\title{
ARQUITECTURA, VEJEZ Y CALIDAD DE VIDA. SATISFACCIÓN RESIDENCIAL Y BIENESTAR SOCIAL
}

\author{
ARCHITECTURE, ELDERLY AND QUALITY OF LIFE. RESIDENTIAL \\ SATISFACTION AND SOCIAL WELL-BEING
}

\section{Luis Arturo Vázquez-Honorato Bertha Lilia Salazar-Martínez}

\author{
LiCenCIATURA EN ARQUiteCtURA, UniversidAD \\ Veracruzana, Xalapa, Veracruz, México \\ RECIBIDO: 7 DE FEBRERO DEL 2010 \\ ACEPTADO: 1 DE SEPTIEMBRE DEL 2010
}

\begin{abstract}
Los autores participaron de manera equiparable en todos los aspectos del escrito. Dirigir la correspondencia al primer autor a Circuito Aguirre Beltrán S/N, Lomas del Estadio. Facultad de Arquitectura. Universidad Veracruzana. C.P. 91000 Xalapa Veracruz, México, o a los correos: luisvh08@yahoo.com.mx y bertha_salazarma@yahoo.com.mx
\end{abstract}

Resumen

La problemática que origina la transición demográfica impacta en todos los países del mundo, y ello conforma una pirámide demográfica invertida. En consecuencia, se hace necesaria la consideración del envejecimiento, en todos los ámbitos, dado que las demandas generadas a partir de este problema no obtendrán las suficientes respuestas en un corto plazo. A partir del enfoque de diferentes puntos de vista, el presente estudio replantea estrategias, en la búsqueda de nuevas respuestas a las demandas de la sociedad que induzca a sus habitantes a no permanecer recluidos, modificando los planteamientos de la contemporaneidad que obligan a la permanencia de sus habitantes en la reclusión de sus residencias, con una mayor influencia sobre los grupos sociales más vulnerables.

Con la comprensión de que la planeación adecuada del espacio habitable demandará en un corto plazo la satisfacción espacial de la población de adultos mayores, el estudio se basa en la identificación dentro del objeto de análisis de los siete principios básicos de diseño universal, permitiendo definir parámetros de diseño para el sistema habitacional desde la perspectiva del anciano. Se realizó el estudio a partir de la obtención de información descriptiva y exploratoria directas llevada a cabo con la toma de una muestra de hasta 500 individuos pertenecientes a las cohortes de 60 años y más de la ciudad de Xalapa, Veracruz, México. En el presente estudio se hace un análisis de variables dependientes que interrelaciona los espacios de la vivienda, del barrio y de la ciudad. Los datos se recopilaron mediante encuestas en asistencias sociales públicas, en visitas domiciliarias y en espacios públicos.

El estudio persigue el beneficio de una integración comunitaria que enriquezca el desarrollo social en ambientes armónicos que reconstruyan el tejido social y que erijan una sociedad más igualitaria. La integración comunitaria incentivará las relaciones, restablecerá los apoyos entre los individuos, en la búsqueda de soluciones a sus problemas cotidianos y ofreciendo con ello una mejor cohesión social.

Palabras clave: Adulto mayor, envejecimiento, diseño universal, hábitat, calidad de vida. 
Vázquez-Honorato \& Salazar-Martínez

\section{Abstract}

The problem giving rise to the demographic transition impact in all countries of the world by instituting a kind of inverted population pyramid. Being necessary the consideration in all the scopes to the aging, since the demand generated from this problem will not get enough answers in the short term. From the approach of different points of view the present study rethinks strategies, in the search for new responses to the demands of society that induces its inhabitants not to remain shut in, changing contemporary approaches which oblige the permanence of their inhabitants in the seclusion of their homes. On having understood that the good planning of the inhabitable space will demand in a short term the spatial satisfaction of the population of major adults, The study is based on the identification, within the scope of analysis of the seven principles of universal design, allowing to define design parameters for the housing system with base in the perspective of the elderly. From the obtaining of descriptive and exploratory direct information, on having realized a sample of up to 500 individuals belonging to the cohorts of 60 years and more, by means of surveys in social public assistances, home visits and in public spaces in the city of Xalapa, Veracruz, Mexico; with an analysis of dependent variables that interrelate to the space of the housing, the neighborhood and the city. In benefit of a community integration, which enriches the social development, in harmonic environments, for reconstruct the social fabric and build a more egalitarian society, which encourages relationships, restoring support among individuals in finding solutions to everyday problems, Offering with it a social cohesion. Keywords: Elderly, aging, universal design, dwelling, quality of life.

\section{Origen del proyecto}

Actualmente, la transición demográfica es un problema que impacta tanto en los países desarrollados como en aquellos en vías de desarrollo. Con el análisis de la situación de los países en vías de desarrollo, es un hecho que la problemática del envejecimiento debe ser tomada en cuenta en todos los ámbitos que, de continuar al ritmo de crecimiento actual, en un mediano plazo, los esquemas de asistencia social, económicos y habitacionales destinados a las personas mayores no serán suficientes, y mucho menos se pensará en la generación de ambientes habitables. De acuerdo con un análisis desde diferentes puntos de vista, y con el objetivo de replantear las estrategias hoy rebasadas, se pretende dar respuesta a las nuevas demandas de nuestra sociedad. Es de suma importancia el estudio de las particularidades propias de los individuos en edad avanzada (su condición económica o social así como de su "debilidad" o "fragilidad") al contar con todas sus capacidades íntegras o disminuidas. Por otra parte, el estudio considera relevantes las relaciones ambientales y psicológicas entre el individuo y su "espacio vivido": del microsistema al mesosistema (es decir, sus ambientes cercanos; p.ej., la casa) y el macrosistema (es decir, lo más lejano; p.ej., la ciudad). El estudio parte de la exploración de la problemática del contexto y de investigaciones que se caracterizan en diversas disciplinas, con preocupaciones por un ambiente que sea física y socialmente mejor. ${ }^{1}$

Existen diversas disciplinas que se articulan en la actualidad mediante investigaciones acerca de un tema específico, lo que permite un mayor conocimiento del tema e impone un esfuerzo creciente del control de la complejidad en el campo de análisis, pero que permite ofrecer una respuesta más exacta, toda vez que así se reconocen todas las variables que intervienen en él.

Los problemas relativos a la "vejez", a "la calidad de la vivienda" y a la "calidad de vida" son analizados por un sinnúmero de profesiones, acentuando la capacidad multidisciplinaria, ya que debiesen interesar al arquitecto no sólo los estudios de los propios colegas, sino también aquellas investigaciones de médicos, psicólogos, asistentes sociales, sociólogos, economistas y juristas, tan sólo por mencionar algunas profesiones. Por consiguiente, es importante agregar que, además de los estudios de carácter científico, existen aportaciones cruciales en el campo de las investigaciones empíricas.

\footnotetext{
1 En el sentido en el que ecología proviene de eco- y de-logía; es decir, ciencia que estudia las relaciones de los seres vivos entre sí y con su entorno; parte de la sociología que estudia la relación entre los grupos humanos y su ambiente, tanto físico como social). Definiciones tomadas del Diccionario de la Real Academia Española, 2010.
} 
Arquitectura, vejez y calidad de vida

La misma amplitud del campo de investigación por considerar ha sugerido hacer algunas elecciones analíticas que se reflejan en la organización práctica de la reseña de acuerdo con la bibliografía (Tacchi, 1995).

Situación demográfica de México

Debido a la importancia que tiene el análisis de los datos estadísticos del país, para la investigación, es imposible soslayar los resultados demográficos que muestran que en el 2005 México contaba con 103 millones de habitantes (Instituto Nacional de Estadística y Geografía [INEGI], 2007), de los cuales alrededor de $8.3 \%$ eran ancianos, con 8500000 adultos mayores con 60 años cumplidos o más; los datos además indican un rezago de seis millones de viviendas y una estimación de la población fuera de los programas de financiamiento habitacional que consideraba a $68 \%$ de ellos, cantidad en incremento al considerar la incorporación de la demanda de más de 750 mil viviendas por año como consecuencia del crecimiento poblacional.

En la actualidad, al tomar en cuenta las proyecciones para los próximos veinte años, se reconoce un aumento sustancial dado que esa población de 103 millones del 2005 (INEGI, 2005) aumentará a 120 millones para el 2030 (INEGI, 2005a) con un requerimiento adicional de 16 millones de viviendas y una cifra de ancianos que, con la transición demográfica, se incrementará a 21240 000, estimando que $17.7 \%$ resultarán ser personas mayores a los 60 años (Consejo Nacional de Población, CONAPO, 2008).

Es perceptible que en un lapso corto la pirámide demográfica de México será de tipo rectangular (INEGI, 2005) como consecuencia de la trasformación de la estructura de edades y dada la disminución de la fecundidad o de la proporción de niños nacidos, cuyo resultado será la contracción de la base piramidal que, a larga, producirá la reducción en el centro de la misma y, en consecuencia, esta tendencia, combinada con la disminución del número de muertes del último grupo etario, constituirá una pirámide demográfica invertida (Del Popolo, 2002).

Con la mención de la vivienda como uno de los indicadores del bienestar y el desarrollo de una nación que incide directamente en el nivel de vida de la población y al que se le considera la infraestructura más demandada, se contempla el espacio donde el ser humano se desenvuelve, convive y proyecta, representando no menos de $60 \%$ del espacio urbano del país, por lo que en una economía como la de México, en la que la disminución del ritmo de crecimiento poblacional denota un incremento del envejecimiento que incide como determinante de las demandas potenciales y efectivas, que en materia de vivienda persiste un rezago importante en el rubro de su suministro con sentido social, o de gestión social del hábitat, toda vez que las políticas en materia de vivienda no han logrado satisfacer la demanda de los grupos más necesitados, definidos en nuestra sociedad como la mayoría de la población y que, aún de manera general, no consideran las tendencias internacionales del diseño universal, ni la búsqueda de una vivienda funcional para todos los grupos etarios, ni las soluciones integrales o las respuestas que conjugan diversas disciplinas.

\section{Hábitat residencial}

Son aún muy pocas las investigaciones realizadas desde el punto de vista del hábitat residencial que consideren parte indisoluble a los espacios habitacional y urbano en la integración de la ciudad.

Es oportuno mencionar que la habitabilidad, en palabras de Serafín J. Mercado, se refiere a:

[...] la relación de los seres humanos con la vivienda, escenario de interacción más antiguo e importante, tanto en lo individual como colectivo y dado que es la unidad social fundamental en los asentamientos humanos que se relaciona estrechamente con la vida familiar[...] (Mercado, 1998).

La importancia de la habitabilidad radica en la valoración que se tiene de un espacio determinado y en la relación que se tiene respecto de la satisfacción de los usuarios, ya sean valoraciones y relaciones objetivas o subjetivas. La valoración puede hacerse en tres niveles: los microsistemas, los mesosistemas y los macrosistemas. Al tener el conocimiento de estos valores puede llegarse a mejorar la calidad de la vivienda y, como resultado, puede llegarse a mejorar la calidad de vida de los individuos, en este caso en particular, de las personas mayores.

Las necesidades que se originan a partir de la demanda de espacio residencial deben ser consideradas de manera prioritaria, toda vez que esta respuesta permitirá colaborar en el impulso a una buena calidad de vida mediante la satisfacción de las 
necesidades de resguardo, protección, habitabilidad, permanencia y pertinencia de estos espacios para los ancianos presentes y futuros. Considerando un escenario sumamente desfavorable dado que en la actualidad no se ha logrado solventar la demanda habitacional y ni siquiera se ha contemplado una respuesta integral que abarque las necesidades de este sector de la población cuya exigencia, según las proyecciones de crecimiento demográfico, serán por mucho mayores de manera inmediata; tendrán repercusiones en la sociedad que aún están por sentirse. Por este motivo, debe fomentarse el mejoramiento de la calidad de vida de los adultos mayores, en primer lugar, por su condición de ancianos y, en segundo, porque es indispensable que puedan y deban convertirse en una fuerza para el desarrollo a través de su "envejecimiento activo" (Regalado Doña, 2002) propiciando condiciones favorables para que los determinantes económicos, sociales, personales, conductuales, de sanidad y servicios sociales así como los entornos físicos favorables se cumplan.
Entornos físicos favorables

Con el estudio de los parámetros para proporcionar a los adultos mayores entornos con las características adecuadas para el desarrollo de sus actividades, se necesita la creación de condiciones políticas, económicas, físicas, sociales y culturales que propicien en la vejez el desarrollo social y el ejercicio de los derechos, deberes y libertades. La sociedad muestra escenarios con un sinnúmero de carencias para vivir esta etapa, tanto en el espacio físico como en los entornos social, político, económico y cultural, que obstaculizan la capacidad de las personas mayores para el logro de cambios significativos en sus condiciones de vida.

Debido a que algunos de los adultos mayores han tenido la oportunidad de atesorar un patrimonio en el transcurso de su vida, existe cierta generalidad de que sean propietarios de la vivienda que habitan, pero ello no quiere decir que, aun cuando tengan resuelto el aspecto habitacional y económico, el patrimonio les brinde respuestas de habitabilidad, seguridad,

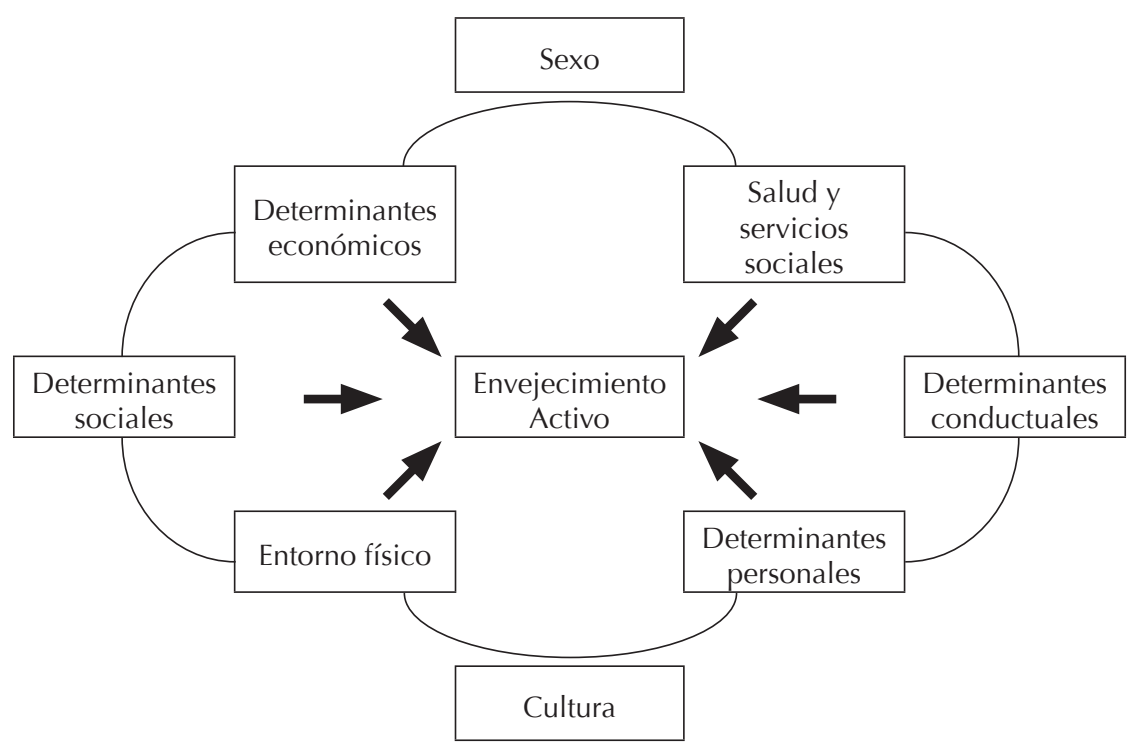

Figura 1. Factores determinantes del envejecimiento activo. La interacción de éstos influye significativamente en la calidad del envejecimiento de las personas. Organización Mundial de la Salud, "Global Age Friendly Cities: A Guide", OMS 2007. p. 10. 
Arquitectura, vejez y calidad de vida

accesibilidad o que contribuya a que su percepción con el entorno sea favorable, disminuyendo así su "calidad de vida". El desafío de la vivienda segura y propicia para las personas mayores implica reconocer, por una parte, la diversidad de necesidades y preferencias de las personas mayores (incluidos la opción y el derecho de "envejecer en cada espacio") $y$, por otra, las situaciones de fragilidad que exigen cuidados y formas especiales para residencia.

Debe garantizarse una vida digna y segura por igual para cualquier grupo etario, sobre todo para el sector más vulnerable (Del Popolo, 2001). Los planteamientos que la modernidad ha definido han estado encaminados a la búsqueda del impulso en una sociedad individualista sin identidad que, en lo referente al desarrollo urbano, se encuentra estrechamente relacionado con la vivienda. Uno de dichos planteamientos es el desarrollo de ciudades extensas que obliga a los habitantes a permanecer recluidos en sus residencias, en especial a los adultos mayores, y a no contar con todos los satisfactores para las actividades que se realizan en el interior de las residencias. Un ejemplo de la carencia de satisfactores es que no exista suficiente espacio en las residencias y que sus habitantes no puedan moverse en ellas, incumpliendo así con la determinante del entorno físico favorable.

Los espacios públicos no están adecuados para recibir a las personas mayores, lo que desalienta en ellos su uso. A fin de que las personas mayores puedan integrarse y ejercer su ciudadanía, especialmente en las área urbanas, se requieren espacios públicos con características físico-espaciales que creen un entorno seguro, accesible y una nueva generación de diseño de este espacio, así como facilidades en el transporte que permitan a las personas mayores ejercer su derecho a desplazarse con autonomía y seguridad, de modo que puedan acceder no sólo a un espacio de intercambio y recreación, sino también a servicios sociales y ejercer sus derechos civiles, políticos, económicos, sociales y culturales. Por este motivo, debemos considerar espacios públicos propicios para todos los grupos etarios, en ciudades más compactas, que permitan el acceso y recorrido por todos los lugares de la ciudad.

Para el disfrute de cada usuario, se requiere que en las áreas construidas se desarrollen polígonos multifuncionales, a fin de eliminar barreras arquitectónicas, polígonos que permitan a cada persona $₫$ sea anciano o niño con distintas habilidades-capacidades $\square$ acceder y utilizar, poder entrar y moverse en ambientes construidos en los que se promueva el "diseño universal" o el "diseño para todos" (Morini, 2007), de tal forma que sirva para satisfacer las necesidades de cada uno de los usuarios.

En la búsqueda de soluciones para el bienestar habitacional de usuarios con capacidades diferentes, el caso de proyectos para ancianos y personas con discapacidad, deben incluirse distintas posibilidades de soluciones tipológicas con un nivel variable de servicios de asistencia, además de pensar en la agregación de las nuevas tecnologías de automatización doméstica y de comunicación, para proveer de un hábitat seguro y confortable en la persecución de dos objetivos: el primero, ofrecer "calidad de vida" para el uso y disfrute de sus habitantes, y el segundo, para evitar accidentes domésticos. Se requiere replantear la vivienda así como la edificación que la alberga y relacionar ésta con el barrio, sin barreras arquitectónicas, ni físicas, ni sensoriales, de tal de modo que sea accesible, cómoda, segura y que permita sobre todo la optimización de tiempos y usos en la movilidad de las actividades cotidianas, que posibiliten la utilización de nuevas tecnologías. Es oportuno mencionar que el hábitat residencial debe ser insertado en forma adecuada en un complejo constructivo y en un contexto urbano que ofrezcan un nivel satisfactorio de apoyos asistenciales, de servicio, de espacios recreativos y de socialización (Landázuri, 2004).

\section{Entornos sociales favorables}

La discriminación por edad se manifiesta de distintas maneras: entre otras, en la falta del reconocimiento hacia las personas mayores, como sujetos a quienes las leyes no tienen contemplado un espacio, víctimas de violencias, etc. Existe la tendencia a presentar a la vejez como una imagen "prototipo", expresada como pasividad, enfermedad, deterioro, carga o ruptura social que, en general, los medios de comunicación mantienen y reproducen. Parte de los activos del capital social acumulado por las personas mayores en el trascurso de su vida son las "redes informales de apoyo social", que son de suma importancia para su bienestar. En el caso de los varones al momento de la jubilación, si no han planeado un nuevo estilo de vida, son altamente vulnerables a perder o reducir sus redes de apoyo. En el caso de 
Vázquez-Honorato \& Salazar-Martínez

las mujeres, las principales dificultades se encuentran en el acceso y disponibilidad de las redes de apoyo formales, sobre todo de la seguridad social (Comisión Económica para América Latina, CEPAL, 2003).

En muchos países europeos, desde la década de 1990, se han realizado investigaciones relativas a las preferencias de los adultos mayores (Morini, 2006), donde el individuo no se considera sólo como "sujeto social colectivo" (Tacchi, 1995), sino alguien con identidad y necesidades propias. Se ha descubierto que los ancianos prefieren envejecer en su vivienda, en lugar de cambiar de residencia o recurrir a instituciones. En estos países, cuando un anciano se transfiere a una institución, se debe a la ausencia de familiares que puedan proporcionarle ayuda. Por este motivo, han fomentado la formación de "redes socio-asistenciales de apoyo" para monitorear y proporcionar ayuda a los adultos mayores o también ocurre la transferencia debido a la falta de soluciones de los espacios que habitan o propiciado por la lejanía de su familia o de los servicios de primera necesidad. Es importante el establecimiento de estas redes para evitar la aparición de otro problema común en nuestra sociedad: la depresión (González-Celis, 2005) en la que el adulto mayor pierde la capacidad para interesarse y disfrutar de las cosas, disminuyendo de tal forma su vitalidad que puede aislarse del resto de la sociedad o, peor aún, si no se llega a atender, puede ocasionarle la muerte. La existencia de investigaciones sobre el tema no es común en México; pero las consideraciones dadas a partir del análisis en nuestra sociedad indican que en ésta no se considera la separación del adulto mayor del seno familiar: se estimula la responsabilidad de atención a las personas mayores, en hijos o parientes cercanos. Otra es la situación cuando las personas mayores son enviadas a espacios asistenciales, situaciones que se da en su mayoría por la falta de una persona que pueda otorgarles las atenciones necesarias.

\section{Referentes}

Basados en los siete principios del diseño universal, se han elaborado propuestas, de las cuales se harán mención de dos: las más significativas y completas.

Como un trabajo de investigación realizado en ciudades de 22 países del mundo, denominado "Ciudades amigables: una guía", a partir de una visión que busca comprender las problemáticas de los grupos más desfavorecidos, con énfasis en los adultos mayores que residen en grandes zonas urbanas de estos 22 países y cuyo fin resulta ser el concretar una respuesta que contribuya a ofrecer mejores alternativas del hábitat construido en el que viven, a partir del desarrollo de un entorno físico y social amigable con el adulto mayor (Organización Mundial de la Salud, OMS, 2007) que induzca al envejecimiento activo y a la búsqueda de la mejora de la calidad de vida de los grupos más desfavorecidos.

El proyecto denominado "Ancianos en casa", elaborado en el 2005 en Europa, se enfoca en la calidad de vida a partir del estudio de cuatro variables: actividades, cualidades, habilidades y recursos, consideradas como claves en la búsqueda del bienestar en la vida de las personas mayores con casa propia (Morini, 2006).

\section{Legislación nacional}

A pesar de que México no ha desarrollado todavía un grado de respuesta necesaria para la sociedad en este rubro, se han contemplado afortunadamente iniciativas plasmadas en las leyes mexicanas en las que se establecen en varios aspectos los derechos de los adultos mayores -como es el derecho a la asistencia sanitaria y a gozar de una seguridad económica- haciendo mención de facilitar el acceso a programas habitacionales para solucionar las necesidades de este grupo etario (Estados Unidos Mexicanos, 2002).

\section{Hipótesis}

Una situación que se adolece en México es la falta de soluciones para los ancianos en materia de espacios habitacionales y de planeación de las ciudades. Es una realidad que las proyecciones de "transición demográfica" generarán cada vez mayores demandas. De ahí la exigencia de propuestas, pues de seguir con la dinámica actual, tales demandas serán difíciles de satisfacer. Deben realizarse estudios que consideren como prioridad la satisfacción de los ciudadanos de la tercera edad. Una aportación de esta investigación es la de crear parámetros de diseño del espacio habitable (vivienda/barrio/ciudad), con la visión de los ancianos. La contribución del estudio vincula la buena planeación del espacio habitable que demandarán en un futuro nuestras ciudades y que estará relacionada positivamente con el nivel de satisfacción espacial de la población en la etapa de adultos mayores. 
Arquitectura, vejez y calidad de vida

Método

Las consideraciones sobre las que se fundamenta el estudio realizado se basa en la obtención de información de tipo descriptiva y exploratoria directa.

\section{Población y muestras}

Se tomó una muestra de hasta 500 individuos, a partir de un análisis correspondiente a las cohortes de 60 años y más. Los datos se recabaron mediante encuestas en asistencias sociales públicas y en visitas domiciliarias y en espacios públicos de la ciudad de Xalapa, Veracruz, México. La duración promedio de las encuestas fue de 20 minutos.

\section{Variables}

En correlación con el grupo etario de estudio, y en referencia al sistema residencial, las variables independientes consideran el espacio de la vivienda (o espacio privado), el espacio del barrio (o semipúblico) y el espacio de la ciudad (o público).

\section{Herramientas}

Los datos recolectados se obtuvieron a partir de la aplicación de una encuesta de 80 reactivos, mediante entrevistas individualizadas, como parte de una tesis doctoral. Ésta se conforma de cinco secciones: a) Características de la vivienda anterior, b) Características de la vivienda actual, c) Características del barrio donde reside, d) Correlación barrio-vivienda y e) Aspectos socioeconómicos y de salud. En la captura de la base de datos se recurrió al programa Excel 2007, y en el análisis estadístico de las variables se utilizó el software STATA versión 9.0.

Resultados

La tabla 1 se refiere a las características de la vivienda actual. Para tener una idea del arraigo e identidad de los habitantes respecto de su vivienda, se les preguntó: "¿Desde qué año residen ahí?". De una población de 489 personas que sí respondieron, $0.20 \%$ (una persona) reside en el periodo que comprende de $1910-1919 ; 1.02 \%$ (5 personas), entre 1920-1929; $2.25 \%$ (11 personas), entre 1930-1939; 6.75\% (33 personas), entre 1940 a 1949; 6.54\% (32 personas), entre $1950-1959 ; 10.63 \%$ (52 personas) entre 1960-1969; $17.59 \%$ (86 personas), entre 1970-1979; $20.25 \%$ (99 personas), entre 1980-1989; 14.93\% (73 personas), entre 1990-1999, y 19.84\% (97 personas) reside allí después del año 2000.

Al categorizar la pregunta " $¿$ Cuantos años tienen de vivir en la vivienda?", se observa que casi $66 \%$ de los adultos mayores tienen más de 20 años de habitar en sus viviendas; $15 \%$ de ellos, entre 10 y 20 años; y 19\%, durante menos de 10 años. Tal situación demuestra que $81 \%$ de las personas de la tercera edad que se entrevistaron tienen un nivel de identificación claro con su vivienda y su barrio por haberla habitado no menos de 10 años.

A la pregunta "¿La vivienda anterior pertenecía a la misma colonia?", de una población de 359 personas que sí respondieron, 69.08 \% declaró que no vivía en la misma colonia (248 personas), 22.84\% afirmó que sí vivía en la misma colonia (82 personas) y $8.08 \%$ mencionó que siempre había vivido en la misma casa (29 personas).

También se cuestionó: “¿Usted ha vivido en la misma región?", y de una población de 366 personas que sí respondió, $70.77 \%$ lo hizo de manera afirmativa (259 personas), y 29.23\% lo negó (107 personas).

Para elaborar el índice de ocupación se utilizaron las variables (número de camas/número de ocupantes). Una población de 429 tiene un índice de ocupación favorable, ya sea mayor o igual: $76.43 \%$ tiene $<1$ por cama (321 personas) y $20.71 \%$ tiene $=1$ por cama (87 personas); en el caso desfavorable, se encuentra que $2.86 \%$ tiene una ocupación $>1$ por cama (12 personas).

Al categorizar estos datos por décadas, puede observarse que el periodo con mayor frecuencia en el que la gente llegó a su vivienda fue en la década de 1960, siendo así que tienen aproximadamente cuarenta años de residir ahí. Con el replanteamiento de la agrupación de los años de residir, se descubre que, en los últimos cuarenta años, 355 personas han tenido movilidad en sus viviendas, lo que representa $71 \%$ de los entrevistados. Ello coincide con los años declarados de vivir ahí. Más de 50\% de los encuestados declaró que no siempre ha vivido en la misma casa, pero sí en la misma región. El resultado de las encuestas indica que $87 \%$ tiene un índice de ocupación favorable; es decir, que no existe hacinamiento en su vivienda; pero, al mismo tiempo, nos indica que puede tener un excedente de espacio desocupado debido a que los hijos ya no se encuentran en casa. 


\section{Vázquez-Honorato \& Salazar-Martínez}

Tabla 1.

Características de la vivienda actual. Población: 500, reactivos: 5.

\begin{tabular}{|c|c|c|}
\hline Características & Frecuencias & Porcentaje \\
\hline \multicolumn{3}{|l|}{$\begin{array}{l}\text { Año en que llegó a vivir a la vivienda } \\
\mathrm{n}=489\end{array}$} \\
\hline $1910-1919$ & 1 & 0.20 \\
\hline 1920-1929 & 5 & 1.02 \\
\hline 1930-1939 & 11 & 2.25 \\
\hline 1940-1949 & 33 & 6.75 \\
\hline 1950-1959 & 32 & 6.54 \\
\hline 1960-1969 & 52 & 10.63 \\
\hline 1970-1979 & 86 & 17.59 \\
\hline 1980-1989 & 99 & 20.25 \\
\hline 1990-1999 & 73 & 14.93 \\
\hline Después del 2000 & 97 & 19.84 \\
\hline \multicolumn{3}{|c|}{$\begin{array}{l}\text { Categorizado el año en que llegó a vivir a la vivienda } \\
\mathrm{n}=489 \\
(1910,2008) \\
4 \pm 3.16 \\
\text { Promedio } 3.16\end{array}$} \\
\hline De 1910-1949 & 50 & 10.22 \\
\hline De 1950-1969 & 84 & 17.88 \\
\hline De 1970-1989 & 185 & 37.83 \\
\hline De 1990-1999 & 73 & 14.96 \\
\hline De 2000-2008 & 97 & 19.84 \\
\hline \multicolumn{3}{|c|}{$\begin{array}{l}\text { Categorizado ¿Cuántos años tiene de vivir en la } \\
\text { vivienda? } \\
\mathrm{n}=489 \\
(0,98) \\
3 \pm 1.22 \\
\quad \text { Promedio } 3.19\end{array}$} \\
\hline $60-98$ & 48 & 9.82 \\
\hline $40-59$ & 84 & 17.18 \\
\hline $20-39$ & 185 & 37.84 \\
\hline $10-19$ & 70 & 14.31 \\
\hline $0-9$ & 102 & 20.86 \\
\hline \multicolumn{3}{|c|}{$\begin{array}{l}\text { Antes de vivir aquí, ¿vivía usted en la misma colonia? } \\
\mathrm{n}=359\end{array}$} \\
\hline Sí & 82 & 22.84 \\
\hline No & 248 & 69.08 \\
\hline Siempre ha vivido en la misma casa & 29 & 8.08 \\
\hline \multicolumn{3}{|l|}{$\begin{array}{l}\text { ¿Vivía usted en la misma región? } \\
\mathrm{n}=366\end{array}$} \\
\hline Sí & 259 & 70.77 \\
\hline No & 107 & 29.23 \\
\hline \multicolumn{3}{|l|}{$\begin{array}{l}\text { Índice de ocupación } \\
\text { Núm. de camas/Núm. de ocupantes } \\
\text { Población } 429 \\
(1,3) \\
1 \pm 0.50 \\
\text { Promedio } 1.26\end{array}$} \\
\hline$<1$ por cama & 321 & 76.43 \\
\hline$=1$ por cama & 87 & 20.71 \\
\hline$>1$ por cama & 12 & 2.86 \\
\hline
\end{tabular}


Arquitectura, vejez y calidad de vida

Tabla 2.

Vidas en pareja y familiar satisfactorias dentro de la vivienda: Población $=500$.

\begin{tabular}{lll}
\hline Características & Frecuencia & Porcentaje \\
\hline ¿Esta vivienda le permite relacionarse & & \\
íntimamente con su pareja? & & 46.60 \\
Sí & 233 & 2.80 \\
No & 14 & 50.60 \\
No tiene pareja & 253 & \\
\hline ¿Esta vivienda le permite desarrollar una & \\
vida de familia satisfactoria? & 353 & 70.60 \\
Sí & 22 & 4.40 \\
No & 125 & 25.00 \\
No aplica (vive solo) & &
\end{tabular}

La tabla 2 muestra los datos a la pregunta " ¿Su vida en pareja es satisfactoria?"; respondió una población de 500 personas, de las cuales 253 personas no tienen pareja $(50.60 \%), 233$ mencionaron que sí pueden relacionarse íntimamente con su pareja $(46.60 \%)$ y 14 personas no pueden relacionarse íntimamente con su pareja $(2.80 \%)$.

A la pregunta "¿Le permite la vivienda desarrollar una vida satisfactoria en familia?", 353 personas contestaron afirmativamente $(70.60 \%), 125$ personas mencionaron que viven solos $(25.00 \%)$ y 22 personas $(4.40 \%)$ mencionaron en forma negativa.

En la tabla 3 se evalúo la calidad del vecindarioentorno vivienda respecto de la calidad de vida y de la relación con los vecinos, por parte de los adultos mayores. A continuación se describen los temas abordados en las preguntas y sus respectivos resultados.

En el rubro de convivencia satisfactoria con los vecinos se tiene: de 500 personas encuestadas, 445 personas $(89.00 \%)$ afirmaron que sí pueden convivir bien con sus vecinos; 55 personas (11.00\%) dijeron que no pueden convivir bien con sus vecinos.

"El hecho de vivir en este barrio lo hace sentir ...": de 424 personas que sí respondieron, 10 personas dijeron que no se sienten nada orgullosos de vivir ahí $(2.36 \%)$; 32 personas (7.55\%) señalaron que se sienten poco orgullosos del lugar; 161 personas $(37.97 \%)$ indicaron que se sienten algo orgullosos de vivir ahí, y 221 personas (52.12\%) afirmaron sentirse muy orgullosos de vivir ahí.

Evaluación de la tranquilidad de su barrio: de 500 personas encuestadas, 280 personas $(56.00 \%)$ calificaron la tranquilidad de su barrio como muy buena;
119 personas (23.80\%), como buena; 67 personas (13.40\%), como regular; 29 personas (5.80\%), como mala, y 5 personas $(1.00 \%)$ calificaron la tranquilidad de su barrio como muy mala.

En cuanto a la evaluación de la seguridad de su barrio se tuvo que de 500 personas encuestadas, 280 personas $(56.00 \%)$ calificaron la seguridad de su barrio como muy buena; 126 personas $(25.20 \%)$, como buena; 65 personas (13.00\%), como regular; 24 personas $(4.80 \%)$, como mala, y 5 personas $(1.00 \%)$ calificaron la seguridad de su barrio como muy mala.

Respecto a la evaluación de la privacidad de su barrio se tuvo que, de 500 personas encuestadas, 275 personas $(55.00 \%)$ calificaron la privacidad de su barrio como muy buena; 134 personas $(26.80 \%)$, como buena; 64 personas (12.80\%), como regular; 20 personas $(4.00 \%)$, como mala, y 7 personas $(1.40 \%)$ calificaron la privacidad de su barrio como muy mala.

Relativo a la evaluación de la belleza de su barrio se tuvo que, de 500 personas encuestadas, 250 personas $(50.00 \%)$ calificaron la belleza de su barrio como muy buena; 114 personas (22.80\%), como buena; 107 personas (21.40\%), como regular; 27 personas $(5.40 \%)$, como mala, y 2 personas $(0.40 \%)$ calificaron la belleza de su barrio como muy mala.

Para la evaluación de la limpieza de su barrio se tuvo que, de 500 personas encuestadas, 247 personas $(49.40 \%)$ calificaron la limpieza de su barrio como muy buena; 135 personas (27.00\%), como buena; 86 personas $(17.20 \%)$, como regular; 27 personas $(5.40 \%)$, como mala, y 5 personas $(1.00 \%)$ calificaron la limpieza de su barrio como muy mala.

En cuanto a la percepción de la calidad del vecindario-entorno-vivienda, la mayoría considera que sí 


\section{Vázquez-Honorato \& Salazar-Martínez}

Tabla 3.

Percepción de la calidad de vida vecindario-entorno inmediato a la vivienda de los vecinos.

\begin{tabular}{|c|c|c|}
\hline Características & Frecuencias & Porcentaje \\
\hline \multicolumn{3}{|c|}{$\begin{array}{l}\text { ¿En esta zona se puede convivir bien con } \\
\text { los vecinos? } \\
n=500\end{array}$} \\
\hline Sí & 445 & 89.0 \\
\hline No & 55 & 11.0 \\
\hline \multicolumn{3}{|c|}{$\begin{array}{l}\text { El hecho de vivir en este barrio lo hace } \\
\text { sentir a usted } \\
n=424\end{array}$} \\
\hline Nada orgulloso & 10 & 2.36 \\
\hline Poco orgulloso & 32 & 7.55 \\
\hline Algo orgulloso & 161 & 37.97 \\
\hline Muy orgulloso & 221 & 52.12 \\
\hline \multicolumn{3}{|c|}{$\begin{array}{l}\text { Evaluación de los siguientes aspectos de su } \\
\text { barrio }\end{array}$} \\
\hline \multicolumn{3}{|c|}{$\begin{array}{l}\text { Tranquilidad } \\
\mathrm{n}=500\end{array}$} \\
\hline Muy buena & 280 & 56.0 \\
\hline Buena & 119 & 23.8 \\
\hline Regular & 67 & 13.4 \\
\hline Mala & 29 & 5.8 \\
\hline Muy mala & 5 & 1.0 \\
\hline \multicolumn{3}{|l|}{$\begin{array}{l}\text { Seguridad } \\
\mathrm{n}=500\end{array}$} \\
\hline Muy buena & 280 & 56.0 \\
\hline Buena & 126 & 25.2 \\
\hline Regular & 65 & 13.0 \\
\hline Mala & 24 & 4.8 \\
\hline Muy mala & 5 & 1.0 \\
\hline \multicolumn{3}{|l|}{$\begin{array}{l}\text { Privacidad } \\
\mathrm{n}=500\end{array}$} \\
\hline Muy buena & 275 & 55.0 \\
\hline Buena & 134 & 26.8 \\
\hline Regular & 64 & 12.8 \\
\hline Mala & 20 & 4.0 \\
\hline Muy mala & 7 & 1.4 \\
\hline \multicolumn{3}{|l|}{$\begin{array}{l}\text { Belleza } \\
\mathrm{n}=500\end{array}$} \\
\hline Muy buena & 250 & 50.0 \\
\hline Buena & 114 & 22.8 \\
\hline Regular & 107 & 21.4 \\
\hline Mala & 27 & 5.4 \\
\hline Muy mala & 2 & 0.4 \\
\hline \multicolumn{3}{|l|}{$\begin{array}{l}\text { Limpieza } \\
\mathrm{n}=500\end{array}$} \\
\hline Muy buena & 247 & 49.4 \\
\hline Buena & 135 & 27.0 \\
\hline Regular & 86 & 17.2 \\
\hline Mala & 27 & 5.4 \\
\hline Muy mala & 5 & 1.0 \\
\hline
\end{tabular}


Arquitectura, vejez y calidad de vida

puede convivir con sus vecinos, ya que sólo $24 \%$ de la población entrevistada manifestó estar expuesto a alguna agresión o a sufrir alguna lesión o caída en el entorno. Las razones por las que no pueden relacionarse son, en primer lugar, por temor a sufrir alguna agresión de algún vecino o grupos pandilleros y, la segunda, se debe a que no pueden salir o moverse libremente. Lo anterior presenta dos opciones: la primera, para propiciar las redes sociales, ya sea de tipo asistencial o de amistad, y la segunda, a fomentar la eliminación de barreras arquitectónicas, así como la propuesta de espacios comunitarios para el uso y disfrute de todos los grupos etarios y, en este caso particular, de los ancianos. Los datos recopilados son muy importantes, ya que aproximadamente el ochenta y nueve por ciento de la población de adultos mayores considera que se siente orgulloso de vivir ahí. El 79.8\% de los adultos mayores considera que la tranquilidad en su barrio está entre buena y muy buena. El $81.20 \%$ de los adultos mayores considera que su seguridad en el barrio está entre buena y muy buena. El $81.80 \%$ de los adultos mayores considera que su privacidad en el barrio está entre buena y muy buena. El $72.8 \%$ de los adultos mayores pondera la belleza de su barrio entre buena y muy buena. El 76\% de los adultos mayores piensa que su barrio es limpio.
En la tabla 4, acerca de la calidad del vecindarioentorno-vivienda respecto de la calidad de vida y relación con los vecinos, se hizo una recopilación de las organizaciones en las que participa, actualmente y con anterioridad, el adulto mayor, así como el porqué no participa en ninguna, en caso de que así sea. Se obtuvo lo siguiente.

De 500 personas encuestadas, 275 personas respondieron que, en organización 1 (véase tabla), 90 personas $(32.73 \%)$ participa en actividades de la Iglesia, 63 personas $(22.91 \%)$ participa en junta de vecinos, 36 personas $(13.09 \%)$ participan en actividades recreativas, 25 personas $(9.09 \%)$ participan en algún club deportivo, 25 personas (9.09\%) participa en la quinta de las rosas, 23 personas (8.36\%) participa en el asilo, 6 personas $(2.18 \%)$ participan en un partido político, 2 personas $(0.73 \%)$ participan en la mesa directiva de padres, 2 personas $(0.73 \%)$ participan en la junta de vigilancia o limpieza; 1 persona $(0.36 \%)$ participa en un sindicato, 1 persona (0.36\%) participa en una asociación de amigos de los animales, y una persona $(0.36 \%)$ participa en un club de jubilados.

Esto quiere decir que $55 \%$ de los ancianos sí participa en alguna actividad; con ello fomenta la creación de redes sociales y mejora sustancialmente su entorno y su calidad de vida.

Tabla 4.

Percepción de la calidad de vida vecindario-entorno inmediato a la vivienda y de los vecinos, participación en organizaciones.

\begin{tabular}{lll}
\hline Características & Frecuencias & Porcentaje \\
\hline $\begin{array}{l}\text { Participa en alguna de las siguientes } \\
\text { organizaciones }\end{array}$ & & \\
\hline $\begin{array}{l}\text { Organización 1 } \\
\mathrm{n}=275\end{array}$ & & \\
\hline Junta de vecinos & 63 & 22.91 \\
Mesa directiva de padres & 2 & 0.73 \\
$\quad$ Iglesia & 90 & 32.73 \\
Club deportivo & 25 & 9.09 \\
Partido político & 6 & 2.18 \\
$\quad$ Junta de vigilancia o limpieza & 2 & 0.73 \\
Actividades recreativas & 36 & 13.09 \\
Sindicato & 1 & 0.36 \\
Quinta de las rosas & 25 & 9.09 \\
Asilo & 23 & 8.36 \\
Amigos de los animales & 1 & 0.36 \\
Club o asociación de jubilados & 1 & 0.36 \\
\hline
\end{tabular}

Journal of Behavior, Health \& Social Issues, vol. 2 num. 2 11-2010 / 4-2011 
Vázquez-Honorato \& Salazar-Martínez

\section{Conclusiones}

Como conclusiones puede mencionarse que en los países latinoamericanos debe considerarse prioritario la resolución de las necesidades de los ancianos, proporcionándoles soluciones inmediatas y adecuadas. En México aún no se le ha dado prioridad a la satisfacción de las necesidades de los ancianos, debido a que no existe una planeación integral entre la ciudad y el hábitat residencial. Deben fomentarse las interrelaciones entre el espacio, el hombre y su entorno para que permitan la flexibilidad de usos, adecuando el espacio a las necesidades en cada una de las etapas de desarrollo del ser humano. El espacio debe ofrecer seguridad, confort y protección, libertad de movimiento a través de los espacios en el interior de la vivienda, una ubicación adecuada insertada en el complejo urbano que considere un nivel satisfactorio de apoyos asistenciales, de servicio, de zonas de esparcimiento y de socialización que faciliten la vida independiente de los ancianos. Para ello deben considerarse las variables prioritarias para el bienestar de las personas mayores a fin de ayudarlos a vivir en su propia casa con la facilitación de actividades, cualidades, habilidades y recursos. Además, debe contemplarse la apropiación de tecnologías que faciliten la realización de sus actividades, dependiendo del nivel económico de los mismos, y la creación de espacios sin disturbios psicológicos al usuario, de tal manera que se beneficie la integración comunitaria, el respeto y la inclusión social de sus habitantes. Ello debe llevarse a cabo con una visión en la que los espacios exteriores, edificios y viviendas posean accesibilidad libre de barreras, que garanticen el bienestar de los usuarios, que les permitan el uso y disfrute plenamente de nuestras ciudades sin límites espaciales donde se integre el espacio exterior (o público) con el interior (o privado) permitiendo la movilidad urbana desde todos los puntos de vista: desde la accesibilidad de transportación, el desplazamiento a través de la red viaria y la visualización clara de los señalamientos o de los espacios abiertos que faciliten su utilización para personas con alguna discapacidad, en todas las épocas del año.

También es necesario hacer conciencia en que lo fundamental para una sociedad es estimular los vínculos familiares y que, a través de éstos, se desarrolle el aprendizaje de valores y retroalimenta- ción de conocimientos adquiridos basados en la experiencia, la búsqueda de incentivos espaciales que faciliten el desarrollo de actividades entre las familias, las posibilidades de multiplicidad de usos que permitan a las familias enriquecer su desarrollo social; pero también que estimulen el aprendizaje del desarrollo ambiental y físico, que entretejan nuestras respuestas las posibilidades de ambientes armónicos, que reconstruyan el tejido social y que erigen una sociedad más igualitaria, capaz de incentivar las relaciones que permitan restablecer los apoyos entre individuos en la búsqueda de soluciones a sus problemas cotidianos, definiendo con ello una cohesión social.

Todo lo anterior debe servir de incentivo para la puesta en práctica de políticas sociales y económicas que permitan el vinculo directo con nuestras sociedades y sus ciudadanos y que a su vez induzcan la generación de espacios sociales a partir del desarrollo de espacios habitables, de acuerdo con respuestas integrales que resuelvan las problemáticas a nivel urbano, del barrio y en la célula mínima urbana sumamente importante la vivienda. Las respuestas integrales deben incrementar la edificación en cantidad, calidad y variedad de propuestas para una población cuya demanda será difícil de satisfacer, pero que a partir de tal demanda se podrán estimular los elementos de cohesión y convivencia en de las comunidades en las que se desenvuelven los adultos mayores.

Los resultados obtenidos en este trabajo han servido de parámetro para la realización de propuestas en materia de habitabilidad para mejorar la calidad de vida de los ancianos en general. Se han obtenido datos interesantes en los aspectos de diseño y constructivos de la vivienda, el barrio y la comunidad, así como la percepción que tiene el adulto mayor en relación con su microsistema y su macrosistema. Después de conocer los indicadores de trabajo, se efectuaron visitas del lugar para encontrar las deficiencias mencionadas por los usuarios en dichas encuestas. Se elaboró un dictamen de los detalles constructivos, y planteando también preguntas al personal de las instituciones, se encontraron los principales problemas a los que se enfrentaban los ancianos y los trabajadores.

Como resultado de esta investigación y el análisis llevado a cabo de los indicadores, se encontró que $75 \%$ de este grupo etario vive en pareja o con algún 
Arquitectura, vejez y calidad de vida

miembro de la familia, y el restante (25\%) vive solo. Este último dato es muy importante, ya que es un sector de la sociedad que debe ser atendido en sus demandas y necesidades, cuya composición como grupo familiar es muy específica, y que incrementa la demanda de vivienda. Existen varias consideraciones en el aspecto del sistema habitacional viviendabarrio-ciudad que deben promoverse, de tal manera que permitan que mediante una política integral. En tal política deberán converger la arquitectura, el desarrollo urbano, la vivienda, la demografía, la sociología, la psicología ambiental, la geografía, la salud y el ambiente, entre otras disciplinas. Es a partir de éstas que es posible definir los fenómenos que permitirán considerar desde tal perspectiva la necesidad de plantearse una ciudad inclusiva que asegure la introducción de una ciudad y un "diseño para todos".

La importancia de cumplir con la satisfacción de los adultos mayores en los tres ámbitos también va en el sentido de desarrollar entornos físicos favorables que contribuyan en gran medida a reducir aspectos psicológicos en este grupo etario, como lo comenta Ana Luisa Gónzalez-Celis (2009):

Uno de los principales problemas de salud mental que afectan a la población mayor de 65 años, es la depresión debido al fuerte impacto psicológico que ocasiona en la vida de las personas, su familia y su entorno.

Para el caso de los ancianos, representa un grupo cada vez más vulnerable debido a los cambios físicos, al deterioro progresivo de la salud, al empobrecimiento social, a la pérdida del trabajo, al abandono del hogar por los hijos, a la muerte de la pareja, entre otros.

\section{Referencias}

Comisión Económica para América Latina, CEPAL. (2003). Estrategia regional de implementación para América Latina y el Caribe del Plan de Acción Internacional de Madrid sobre el Envejecimiento. Santiago de Chile. CEPAL.19 al 21 de noviembre de 2003. 22-23.

Comisión Nacional de Vivienda, CONAPO. (2008). Programa Nacional de Vivienda 2007-2012, hacia un desarrollo habitacional sustentable. México.

Del Popolo, F. (2001). Características demográficas y socioeconómicas de las personas de edad en
América Latina. Ed. Naciones Unidas, CEPAL/ CELADE, Santiago de Chile. 15-70.

Del Popolo, F. (2002). Los problemas en la declaración de edad de la población adulta mayor en los censos. Ed. Naciones Unidas, CEPAL/CELADE, Santiago de Chile. 38-45.

Gónzalez-Celis, A. (2005). Los adultos mayores: un reto para la familia. En: Dinámica de la familia. (pp. 127-129). México: Pax.

Gónzalez-Celis, A. (2009). Composición factorial del inventario de depresión de Beck en ancianos mexicanos. Journal of Behavior, Health \& Social Issues. $1^{\circ}$ de mayo, 15-28, disponible vía: http:// dx.doi.org/10.5460/jbhsi.v1.i1.02

Instituto Nacional de Estadística y Geografía, INEGI. (2005). Conteo de población 2005. México.

Instituto Nacional de Estadística y Geografía, INEGI. (2005a). Proyecciones nacionales 2005-2051. México.

Instituto Nacional de Estadística y Geografía, INEGI. (2007). México hoy, México.

Landázuri, A., \& Mercado, S. (2004). Algunos factores físicos y psicológicos relacionados con la habitabilidad interna de la vivienda. En: Medio Ambiente y Comportamiento Humano, 5 (1 y 2), Ed. Resma.

Ley de los derechos de las personas adultas mayores de México. Estados Unidos Mexicanos. (2002).

Mercado, S. J. (1998). La vivienda: Una perspectiva psicológica. En: J. Guevara, A. M. Landázuri y A. Terán. "Estudios de Psicología Ambiental en América Latina. México". (pp. 141-153). México: Coedición: BUAP-UNAM-CONACYT.

Morini, A. (2006). Progetto Elderathome, La ricerca europea "anziani a casa propria. Asistenza Anziani/gennaio. (p. 20). Roma: Istituto per le Tecnologie della Costruzione.

Morini, A. (2007) Progetto LIVINGALL. Asistenza Anziani/agosto. (pp. 9-11). Roma: Istituto per le Tecnologie della Costruzione.

Organización Mundial de la Salud, OMS. (2007). Ciudades Amigables. Francia, 10-15.

Organización Mundial de la Salud; Grupo Orgánico de Enfermedades No Transmisibles y Salud Mental. Departamento de Prevención de las Enfermedades No Transmisibles y Promoción de la Salud Envejecimiento y Ciclo Vital. Revista Especial Geriátrica, Gerontológica, 37-40. 


\section{Vázquez-Honorato \& Salazar-Martínez}

Real Academia Española (2010). Diccionario de la lengua española, vigésima segunda edición.

Regalado, P. J. (2002). Envejecimiento activo: un marco político (traducción).
Tacchi, E. M. (1995). Láanziano e la sua casa. (17-18). Italia: FrancoAngeli. 